\title{
ULTRASONIC DUPLEX SCANNING IN ATHEROSCLEROTIC DISEASE OF THE INNOMINATE, SUBCLAVIAN AND VERTEBRAL ARTERIES. A COMPARATIVE STUDY WITH ANGIOGRAPHY
}

\author{
R. G. A. Ackerstaff, H. Hofnkvei d and J. M. Siowikowski \\ Department of Clinical Neurophysiology, St. Antonius Hospital, Utrecht, The Netherlands \\ F. L. Moll and B. C. Eikelboom \\ Department of Vascular Surgery, St. Antonius Hospital, Utrecht, The Netherlands \\ and \\ J. W. LUDWIG \\ Department of Radiology, St. Antonius Hospital, Utrecht, The Netherlands
}

(Received 18 May 1983; in final form 27 September 1983)

\begin{abstract}
Ultrasonic duplex scanning of the vertebral artery has a sensitivity of 0.80 and a specificity of 0.83 for the detection of an obstructive lesion of $50 \%$ or more at the site of the ostium. For the subclavian arteries these values are respectively 0.73 and 0.91 . For both vessels the test has a very high negative predictive value of respectively 0.96 and 0.97 . Therefore duplex ultrasound scanning is a reliable test in screening patients suspected of multi-level atherosclerotic disease of the extracranial cerebral vessels. The problem remains in classifying the degree of stenosis. In the case of the vertebral artery the sample volume of the pulsed Doppler is usually too large in relation to the vessel diameter. In the case of the subclavian and innominate artery one of the main problems is the range of the pulsed Doppler system. A subclavian steal syndrome is easily diagnosed without any special test.
\end{abstract}

Key Words: Ultrasonics, Duplex-scanning, Blood flow velocity, Vertebral artery, Subclavian artery, Innuminate artery.

\section{INTRODUCTION}

The adverse clinical effects of atherosclerotic plaques in the carotid or vertebral arteries are manifested by either embolization from the site of the lesion or through reduction of blood flow. The most common symptoms of cerebral ischemia of the carotid territory are amaurosis fugax, transient ischemic attack and stroke. The appearance of certain transient neurological deficits such as vertigo, intermittent deafness, blurred vision, ataxia, syncope and drop attacks may be attributed to transient ischemia occurring within the vertebrobasilar system. These symptoms are commonly accompanied by occipital headache and are usually termed the vertebrobasilar arterial insufficiency syndrome.

An early clinical diagnosis of brain stem and occipital lobes ischemia is of prime importance (Rosenthal et al., 1978; Cormier et al., 1976; Khodadad et al., 1978). Unfortunately angiography has its limitations. Complications from intraluminal manipulation of the catheter and allergy to the contrast material cannot be ncglccted. The amount of contrast material which may be injected in one examination is limited by the potential loss of renal function (Hessel et al., 1981). This is also true for the recently developed digital intravenous subtraction angiography. Preangiographic information about the likely location of the atherosclerotic plaques is very helpful.

Several non-invasive techniques can offer such information. The majority of these methods focus at the carotid bifurcation or at the orbital region. The study of the vertebral artery is for several reasons much more difficult than the carotid bifurcations. In the event of atherosclerotic disease of the vertebral artery the ostium is involved in more than $50 \%$ of the cases (Thevenet, 1979). Therefore the site of most interest is the origin of the vertebral artery from the subclavian artery.

Continuous-wave Doppler sonography is a reliable method for detecting severe subclavian stenosis and occlusion. The study of the origin of the vertebral artery is, however, usually impossible by this method. For this reason, using a continuous wave Doppler, the vertebral arteries are usually investigated more distally, where the artery loops around the atlas. However, without the use of special tests it is not possible to positively determine the direction of flow, since one does not know which part of the atlas loop is insonated by the ultrasonic beam. Moreover there is often asymmetry in the vertebral artery diameters 
and a differentiation between an occlusion and a hypoplasia of the artery can be difficult.

Several authors have examined the vertebral artery with a continuous-wave or single gate pulsed Doppler imaging system (Spencer et al., 1977, White et al., 1980). In most cases it is impossible to identify the prebony part of the vertebral artery with certainty with these techniques. Wood et al. (1980) describe a technique for identifying the origin of the vertebral artery with a multigated pulsed Doppler imaging system. In their study, insufficient patients have undergone contrast angiography to allow data on diagnostic accuracy.

The duplex concept is based upon the ability to define the walls of vessels within a two-dimensional ultrasonic image and employs a pulsed Doppler to evaluate the blood velocities within the visualized artery. With this system it is possible to study the anatomy of the vessels and in particular the course of the proximal part of the vertebral artery and the location of its ostium. In most cases it is possible to measure the velocity of flow near the origin of the vertebral artery. In addition the direction of blood flow can be ascertained and the diagnosis of vertebral artery disease can be suspected by hearing turbulence just distal to the site of disease. By means of spectral analysis of the Doppler signal one can identify several categories of stenosis. In skilled hands it is even possible to examine the vertebral artery in the bony canal as well as the more proximal part of the subclavian or innominate artery. This study will review the author's results with the use of the ultra- sonic duplex scanner in the evaluation of 82 patients who have undergone contrast arteriography.

\section{METHODS AND MATERIALS}

The examination of the innominate, subclavian and vertebral arteries is a part of a more general ultrasonic duplex scanning of the extracranial cerebral vessels. The real time $B$-mode image is generated by three $5-\mathrm{MHz}$ ultrasonic transducers mounted on a rotating drum encased in a water-filled head ( $\Lambda$.T.L. mark 5). During scanning, changes of velocity of flow are detected with a single gate, variable range, $5-\mathrm{MHz}$ pulsed Doppler. The beam axis of the Doppler is represented on the image by an enhanced white line; the point from which flow is sampled is represented by a white dot (Fig. 1). Both the $B$-mode image and the directional Doppler signal are recorded on tape for review and processing of the velocity signal. A digital fast Fourier transform spectrum analyzer (Nicolet UA-500 A) is used to process the Doppler signal. A hard copy is produced on light-sensitive paper to display the frequencies, the directional characteristics and the amplitude in shades of grey of the Doppler signal.

During the examination the patient lies in a relaxed supine position with his head on a small pillow. The study is started in the supraclavicular fossa by visualizing the innominate or subclavian artery. These vessels are followed in a distal direction until the vertebral artery is found. To prevent mistakes it is important to realize that, in contrast to the thyrocervical trunk, the vertebral artery has a typical

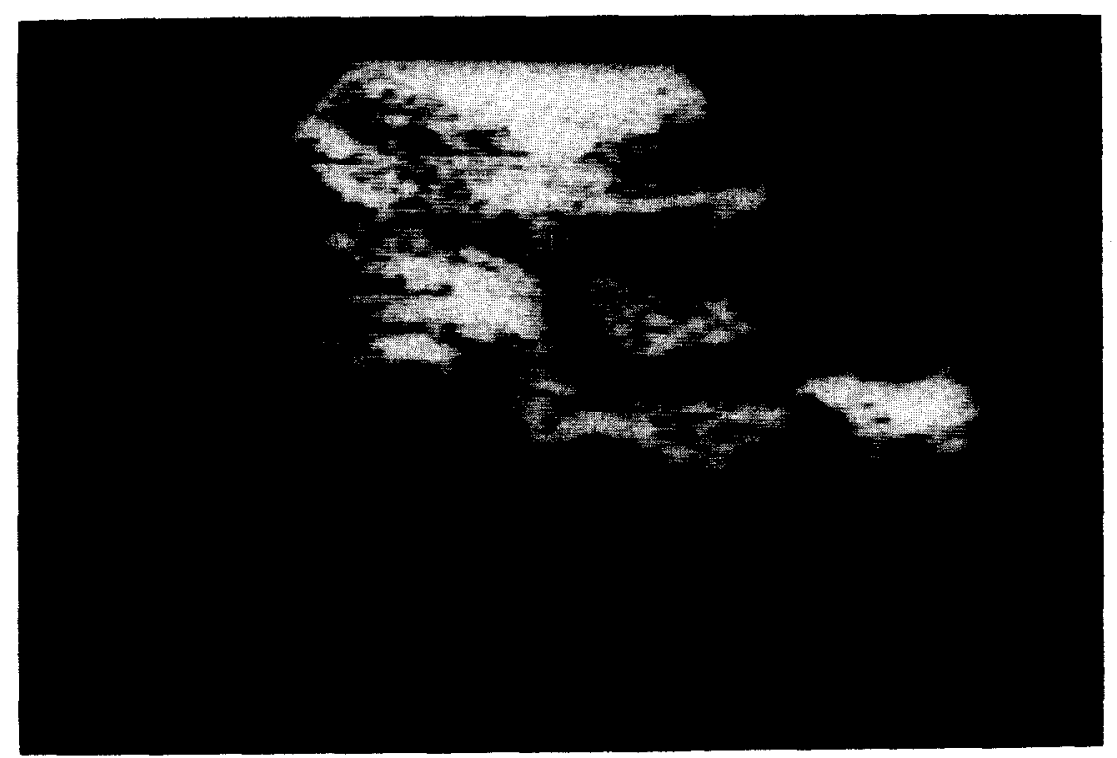

Fig. 1. A B-mode image of the subclavian and the most proximal part of the vertebral artery. The subclavian artery is on the right side and partially seen in a transverse plane. The vertebral artery is visualized along its longitudinal axis. The sample volume is represented by the white spot and is placed in the second curve of the vertebral artery. 
course with a flat curve just distal to its origin. The location of the ostium and the course of the artery are carefully noted. In most cases it is possible to find a vertebral artery without any problem. Usually the axis of the vessel relative to the Doppler beam is less than $90^{\circ}$ and a reliable Doppler measurement is possible. Sometimes in spite of many efforts the axis of the vessel remains perpendicular to the Doppler beam and a Doppler measurement is impracticable.

Routinely three or four Doppler measurements are performed on each side; the innominate artery, the subclavian artery, and the proximal and distal vertebral artery.

In some cases the vertebral artery is visualized only a few centimeters distal to its origin from the subclavian artery. It is very well discerned and the direction of flow can be detected, but the ostium itself lies too deep. Thus, it may be out of the range of the pulsed Doppler device and it is not possible to measure the velocity of flow near the ostium.

The operator measures the angle of the Doppler beam relative to the axis of the vessel of interest. In contrast with the examination of the carotid arteries it is not always possible to obtain an angle of approx. 60 , because of anatomic variations. To avoid detection of velocities and eddies close to the vessel wall, in large vessels such as the subclavian or the innominate artery, the sample volume is placed near the center stream. Under normal conditions with a laminar flow during systole the red blood cells are moved forward with more or less the same velocity. So the Fourier transform of the Doppler signal shows a narrow band of frequencies with a clean window below the systolic peak (Figs. 2 and 3). With regard to the much smaller vertebral arteries one always obtains reflections from the slowly moving red blood cells near the vessel wall. This results in an increase of the spectral width and the window below the systolic peak is filled in.

This phenomenon is called spectral broadening. In ultrasonic duplex scanning of the carotid arteries this is a sign of minor wall abnormalities (Ackerstaff et al., 1982; Breslau, 1982; Fell et al., 1981). However, in our experience it is a normal finding in vertebral arteries.

The spectral changes evaluated include the peak frequencies, the degree of spectral broadening during systole and the direction (forward, zero or reverse flow) of the signal. If with ultrasonic duplex scanning a Doppler measurement is possible, one is always certain about the direction of blood flow. Due to the low peripheral flow resistance in the vertebral artery which supplies the brain there is a forward flow during the whole cardiac cycle. Finding reversed flow in the vertebral artery is a sign of severe pathology.
The cause of flow reversal is a hemodynamic significant stenosis of the subclavian artery proximal to the ostium of the vertebral artery. In vertebral arteries, flow reversal is sometimes cardiac cycle dependent (von Reutern et al., 1978).

According to the characteristic spectral changes of the Doppler signal recorded in the vertebral artery, three categories can be defined.

(a) Normal. Physiological antegrade direction of flow, peak frequencies less than $4 \mathrm{kHz}$ and moderate spectral broadening during systole without striking turbulence (Fig. 4).

(b) Abnormal. Physiological antegrade direction of flow, peak frequencies more than $4 \mathrm{kHz}$, an increased spectral broadening and striking turbulence during systole (Fig. 5).

(c) Total occlusion. Usually no image of the vertebral artery is acquired and a Doppler measurement is impracticable.

For the innominate and subclavian artery the criteria are somewhat different. The flow patterns from these arteries shows a steep systolic wave and after the onset of diastole, a brief period of flow reversal. During later diastole there is no significant flow present in the subclavian artery. The innominate artery, however, usually shows a forward flow during diastole (Fig. 2). Under normal conditions the peak frequencies obtained in the arch vessels are often greater than $4 \mathrm{kHz}$. If the sample volume is placed near the center stream the recorded Doppler signals are clean. With increasing stenosis there is more and more turbulence and one will hear a harsh Doppler signal. If an obstructive lesion is located proximal in the innominate or in the subclavian artery it is not always possible to place the sample volume near the stenotic area. The outline of the Fourier transform is irregular a few centimeters downstream to a significant stenosis and the spectrum often reveals loss of frequencies (Fig. 6). In the event of a total occlusion of the subclavian artery with a subclavian steal one finds a low systolic flow velocity in the post-stenotic segment of the subclavian artery. A remarkable asymmetry of the Doppler signals is very important for the judgement of abnormalities of this kind in the subclavian arteries.

From May 1981 to November 1982, 900 ultrasonic studies were performed. Of these patients 65 men (mean age $63 \mathrm{yr}$ ) and 17 women (mean age $58 \mathrm{yr}$ ) underwent four-vessel angiography. In all patients the Seldinger technique was used with a visualization of the aortic arch and arch vessels, usually in two planes. Besides biplane carotid arteriography there was a semi-selective study of the vertebrobasilar system, sometimes with cinematography of the proximal part 


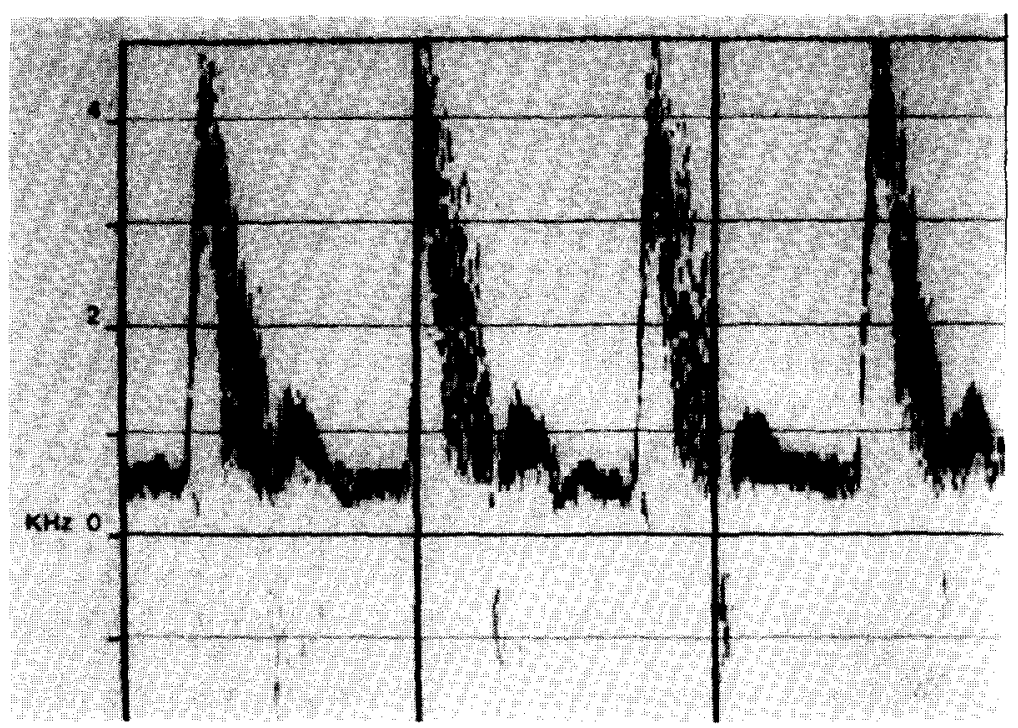

Fig. 2.

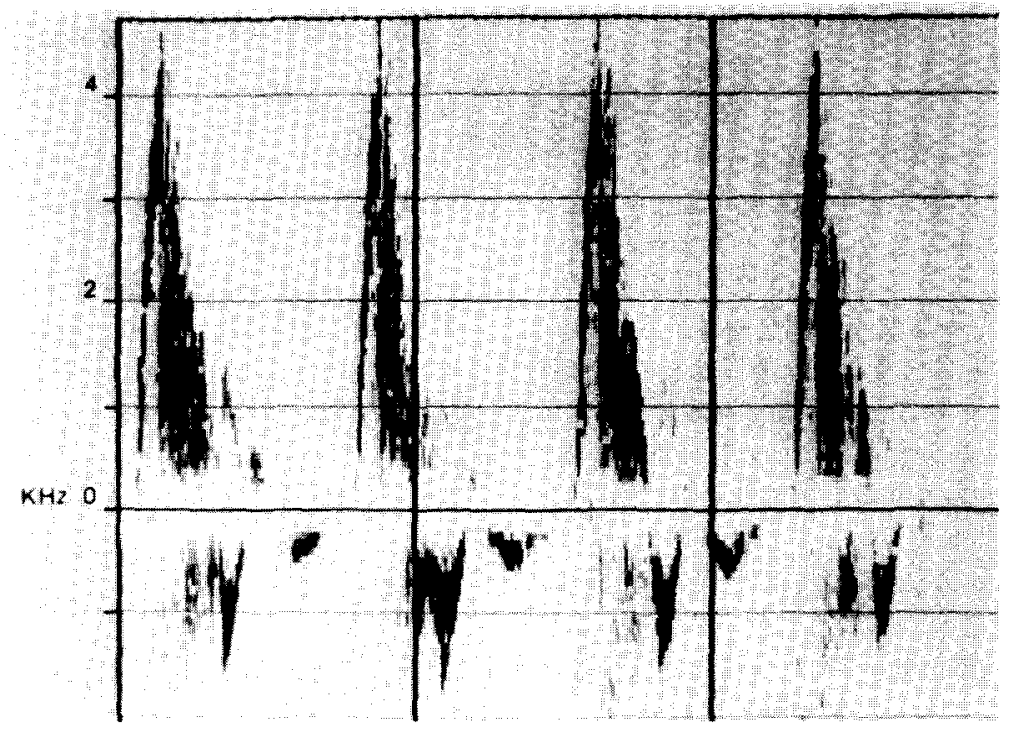

Fig. 3.

Figs. 2 and 3. The Fourier transform of the Doppler signals of the innominate and subclavian arteries respectively. Note the forward flow during diastole in the innominate artery.

of the vertcbral artery. For a proper estimate one has to realize that in the event of a normal ostium of the left vertebral artery a selective study of the right ostium was not routinely performed. The radiologists were unaware of the results of the noninvasive tests. They measured the degree of stenosis by comparing the smallest diameter at the site of the lesion with the diameter just distal to it.

Seventy-nine patients were referred for evaluation of suspected atherosclerotic disease of the extracranial cerebral vessels. In one patient the diagnosis was fibromuscular dysplasia, in one patient moyamoya disease and in one patient Takayasu's disease.

\section{RESULTS}

In this study we had a complete radiological documentation of the aortic arch, the innominate and subclavian arteries and of the vertebrobasilar system in 82 patients. In one patient the angiographic study was performed only on the left side.

\section{Vertebral artery}

Of the 163 vertebral arteries in this study a reliable radiologic estimate of the origin was not possible in 46 cases.

In 31 of the remaining 117 sides we could not identify the vertebral artery with the ultrasonic duplex scanner. In 14 instances we found a vertebral artery, 


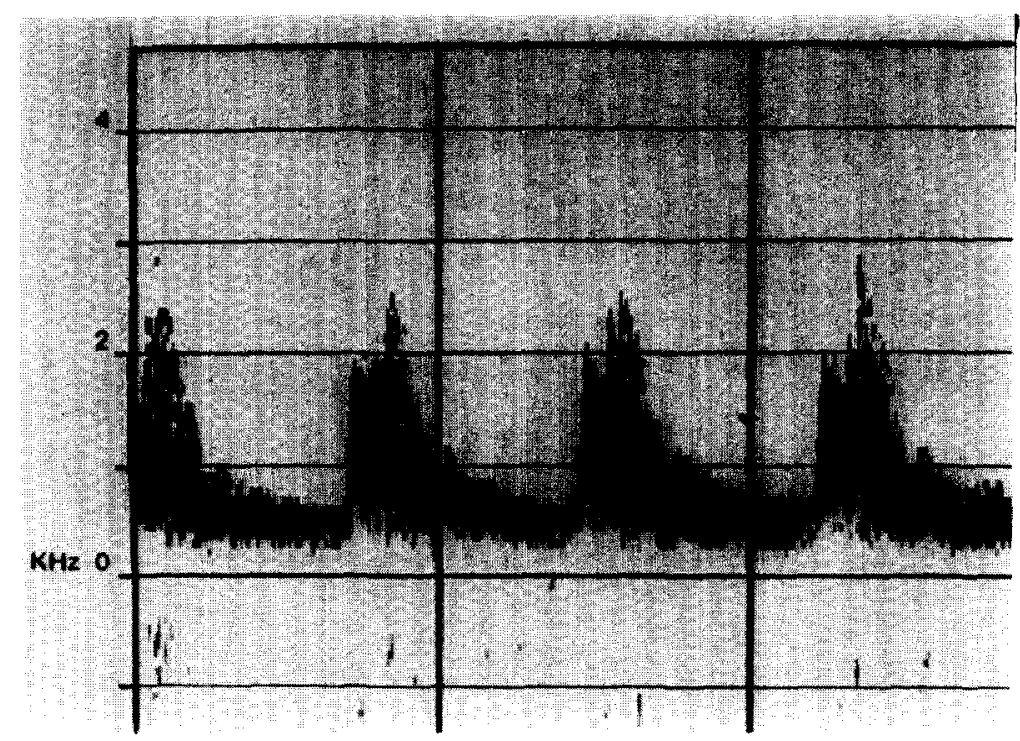

Fig. 4. The Fourier transform of the Doppler signals of a normal vertebral artery. As the sample volume is large relative to the vessel diameter, there is usually spectral broadening during systole.

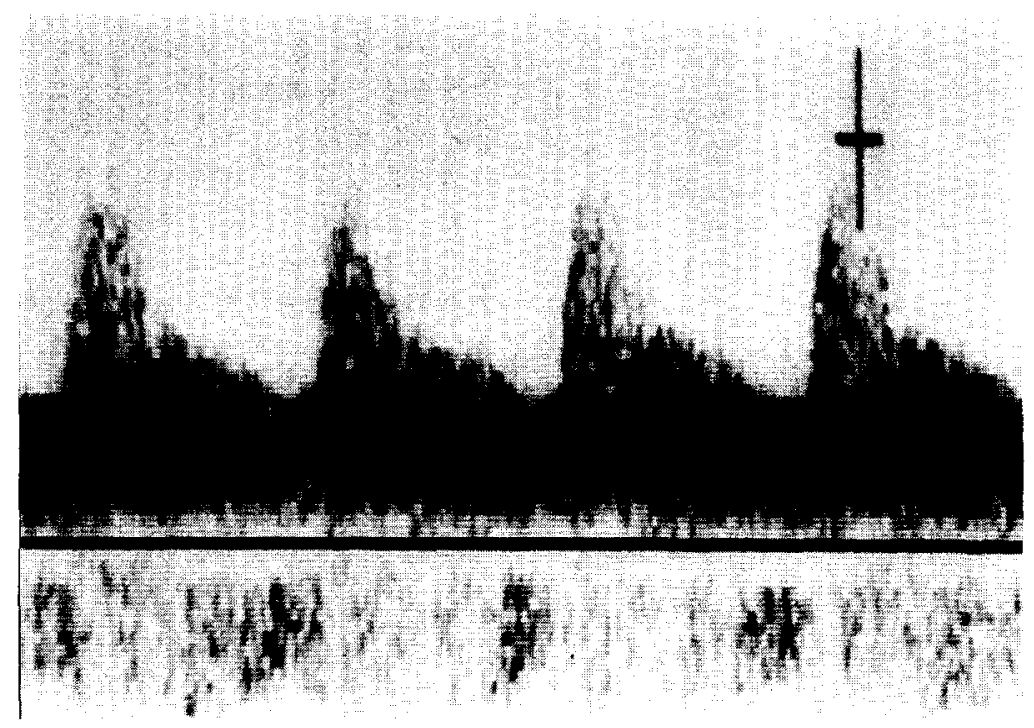

Fig. 5. The Fourier transform of the Doppler signals of a vertebral artery with a significant stenosis at the site of the ostium. Note the increased peak frequencies (cross: $5.3 \mathrm{kHz}$ ) and spectral broadening.

but the results of duplex scanning did not permit a decision concerning the state of the ostium. Therefore we were able to compare the data from contrast arteriography of the ostium of 72 vertebral arteries with the data of ultrasonic duplex scanning. The results of this comparison are shown in Table 1. Of the 49 vertebral arteries, which showed no abnormalities at their origin, 45 were correctly identified. In four cases the duplex study suggested a stenosis. Of the 23 arteries with an angiographically determined obstructive lesion the results of the duplex scanning were in agreement in 18 cases. In five cases there was a false negative result. Only two of these five arteries had a stenosis of $50 \%$ or more. The sensitivity, the specificity, the positive predictive value and the negative predictive value for identifying a stenosis of $50 \%$ or more were calculated (Table 4). In this study we could not identify the vertebral artery with the duplex scanner in 31 instances. The results of the contrast arteriographic examination of these 31 arteries are shown in Table 2 . In one case the artery remained invisible during the whole radiologic study and the 
Table 3. Correlation of duplex results and contrast arteriographic results for the subclavian artery

\begin{tabular}{|l|cccc|}
\hline $\begin{array}{l}\text { Stenosis } \\
\text { on } \\
\text { ANGIO- } \\
\text { GRAPHi }\end{array}$ & normal & abnormal & occlusion & total \\
\hline $0 \%$ & 112 & $3+3^{+}$ & 118 \\
$1-49 \%$ & 9 & 6 & 15 \\
$50-99 \%$ & 4 & 6 & 1 & 10 \\
$100 \%$ & 125 & 22 & 1 & 5 \\
\hline total & & $4^{++}$ & 148 \\
\hline
\end{tabular}

+ In three patients turbulent flow in the subclavian artery is probably the result of a stenosis of the innominate artery.

++ This concerns four patients with an occlusion of the subclavian artery and a stcal from the vertebral artery or from the thyrocervical trunk.

15 arteries with a mild stenosis ( $1-49 \%$ dia. reduction) and in four of the ten arteries with a marked stenosis (50-99\% dia. reduction) the results of the duplex study were estimated as normal. In this study there were five occluded subclavian arteries. In three of these five arteries there was a subclavian steal from the ipsilateral vertebral artery. In another artery there was a steal from the ipsilateral thyrocervical trunk. These four cases with a subclavian steal were correctly identified. The last occluded subclavian artery was identified as well, but we failed to find the ipsilateral vertebral artery. The sensitivity, the specificity, the positive predictive value and the negative predictive value for identifying a stenosis of $50 \%$ or more and occlusion were calculated (Table 4). The innominate artery usually lies rather deep and therefore the examination of this vessel was not routinely carried out at the beginning of this study. As Table 5 shows sometimes a turbulent and rounded Doppler signal in the subclavian or in the common carotid artery was a sign of an obstructive lesion in the innominate artery.

\section{DISCUSSION}

Atherosclerosis is a diffuse process. Recently Hennerici et al. (1981) showed that in patients with systemic atherosclerosis the incidence and extent of asymptomatic extracranial arterial disease is high. In about a quarter of the patients with peripheral vascular and asymptomatic extracranial disease, the innominate-subclavian-vertebral system was involved in the atherosclerotic process. The involvement of the vertebrobasilar system seems to be important in increasing the stroke risk of patients with obstructive lesions of the carotid artery (Sindermann, 1967; Hennerici et al., 1980).

In patients with vertebrobasilar arterial insufficiency clinical and pathological studies have shown that atherosclerosis of the vertebral and basilar arteries was, beside other causes as cervical spondylosis, generally present (Sheehan et al., 1960, Naritomi et al., 1979). With progression of the atherosclerotic process of the innominate or subclavian artery a reverse flow in a vertebral artery may develop. If a steal from a vertebral artery gives rise to clinical symptons of vertebrobasilar arterial insufficiency depends on the status of other extracranial vessels and the collateral circulation.

Therefore, for obvious reasons in many patients with atherosclerosis of the extracranial and arch vessels the symptons of ischemia of the carotid territory may interfere with the clinical picture of vertebrobasilar arterial insufficiency. Reliable information about the status of the innominate, subclavian and vertebral arteries is important in that case, determining the risk of systemic atherosclerosis in symptomatic and asymptomatic patients. Since angiographic examination is usually confined to selected patients, non-invasive techniques have been developed for the detection of flow abnormalities in the vertebrobasilar system. It has been reported that use of Doppler techniques provides information on the adequacy of the posterior brain circulation (Büdingen et al., 1977; Keller et al., 1976). Many Doppler

Table 4. The calculated values of the sensitivity, specificity, positive predictive value and negative predictive value for identifying a stenosis of $50 \%$ or more of respectively the vertebral (ostium) and subclavian artery

\begin{tabular}{|c|c|c|c|c|}
\hline YISSEL & sensitivity & specificity & $\begin{array}{c}\text { positive } \\
\text { predictive } \\
\text { value }\end{array}$ & $\begin{array}{l}\text { negative } \\
\text { predictive } \\
\text { value }\end{array}$ \\
\hline $\begin{array}{l}\text { vertebral } \\
\text { artery }\end{array}$ & 0.80 & 0.83 & 0.45 & 0.96 \\
\hline $\begin{array}{l}\text { subclavian } \\
\text { artery }\end{array}$ & 0.73 & 0.91 & 0.46 & 0.97 \\
\hline
\end{tabular}


Table 5. Correlation of duplex results and contrast arteriographic results for the innominate artery

\begin{tabular}{|c|c|c|c|c|}
\hline \multirow{2}{*}{ So } & \multirow{2}{*}{$\begin{array}{l}\text { stenosis on } \\
\text { angiography }\end{array}$} & \multicolumn{3}{|c|}{ resulls of duplex scantuing } \\
\hline & & innominate & subclavian & common carotid \\
\hline 1 & 25 & - & turbulence & normal \\
\hline 2 & 30 & - & $\begin{array}{l}\text { abnormal } \\
\text { turbulence }\end{array}$ & turbulence \\
\hline 3 & 35 & - & $\begin{array}{l}\text { abnorma1 } \\
\text { turbulence }\end{array}$ & normal \\
\hline 4 & 35 & - & normal & rounded \\
\hline 5 & 45 & - & - & normal \\
\hline 6 & 45 & turbulence & turbulence & rounded \\
\hline 7 & 50 & - & normal & norma 1 \\
\hline 8 & 50 & - & normal & rounded \\
\hline 9 & 70 & - & turbulence & turbulence \\
\hline 10 & 30 & nornal & normal & norma 1 \\
\hline 11 & 75 & - & normal & normal \\
\hline
\end{tabular}

techniques are indeed of substantial help in making an individual estimation of the severity of disease of the innominate-subclavian-vertebral system, but they do not as yet yield quantitative information. A subclavian steal phenonemon can be reliably diagnosed by this non-invasive technique, but in contrast to the vertebral system a differentiation of the underlying vascular disease is not possible. The main problems are the investigation of the proximal part of the vertebral artery and the differentiation of severe stenosis, occlusion, hypoplasia and aplasia (von Reutern et al., 1976, Hennerici et al., 1981). Ultrasonic duplex scanning with spectral analysis of the Doppler signal is a very reliable technique for the detection of carotid atherosclerosis (Fell et al., 1981; Breslau, 1982; Ackerstaff et al., 1982). To date no reports are available about the accuracy of duplex ultrasound scanning of the innominate, subclavian and vertebral arteries. The $B$-mode image of the arteries is an anatomical guide by which one is informed about the exact location and course of the vessels of interest. The investigation of the proximal part of the vertebral artery is usually not a problem and an accurate Doppler measurement just distal to the ostium or at other selected sides is possible. Although some diagnostic significance is attached to the ultrasonic image, detection of an abnormal Doppler signal is the primary criterion used in the diagnosis of an obstructive lesion. Spectral analysis of the Fourier transform of the Doppler signal allows a classification to be made of the severity of disease. In this study we have used a medium focus transducer. The beam width of this transducer is at its narrowest between the 35-55 mm range (Knox et al., 1982). Therefore the medium focus scan head is appropriate for the investigation of the innominate, subclavian or vertebral arteries, usually lying further than $30 \mathrm{~mm}$ from the transducer. Additionally, the size of the sample volume, relative to the size of the vessel being studied, has a considerable effect on the spectra of the velocity wave form. Relative to the vessel diameter the sample volume is large for the vertebral artery. Under normal conditions this results in a greater distribution of velocities and a broadening of the spectral width. Relative to the vessel diameter of the innominate or subclavian artery the sample volume is usually very small. If under normal conditions the sample volume is placed near the center stream the wave forms from these vessels show a narrow band of frequencies. Lesions which compromise the lumen produce turbulence and in general the degree of turbulence is proportional to the degree of stenosis.

The results of this study show that ultrasonic duplex scanning of the proximal part of the vertebral 
artery is a reliable test. In the beginning one of the main problems was to find the vertebral artery. In this study we failed in 31 instances. Now with more experience this seldom happens. Table 2 shows that of the 31 instances in which no vertebral artery was found with the duplex scanner there was an occlusion of the vertebral artery in $26 \%(8 / 31)$. In one case the cause was probably a congenital absence, in one case an abnormal origin from the aortic arch and in three instances a marked hypoplasia. The problem is that, in contrast with the ultrasonic examination of the carotid bifurcation, it is usually not possible to image the vertebral artery in the event of an occlusion. An important reason for this may be the limited capabilities of the available scan heads. Table 1 shows that of the 49 angiographically normal vertebral ostia the duplex study suggested a stenosis in only four cases. Another problem was the classification of the degree of stenosis. Relative to the diameter of the vertebral artery the sample volume of the scan head was too large. The detection of minor obstructive lesions by use of spectral broadening is not possible at the present time. Nevertheless, of the five arteries in which the duplex scanning gave a false negative result there was a haemodynamic significant lesion in only two cases. For the detection of an obstructive lesion at the site of the ostium of the vertebral artery of $50 \%$ or more the sensitivity and the specificity were respectively $0.80(48 / 60)$ and $0.83(10 / 12)$. The predictive value of a normal test result was remarkably high $(0.96,48 / 50)$. The predictive value of a positive test result, however, was very low $(0.45,10 / 22)$. In the 163 vertebral arteries investigated in this study in 14 cases it was impossible to make a decision upon the patency of the ostium with the duplex scanner. The finding of an artery by the technician and the scanning of a more distal part of the vessel was not a problem, but the ostium itself lay too deep. On the other hand a reliable radiologic estimate of the origin of the vertebral artery was impracticable in 46 cases. This high number was partly the result of the followed radiological procedure.

At this moment we use ultrasonic duplex scanning of the vertebral artery as a screening test in patients suspected of multi-level atherosclerotic disease of the extracranial cerebral vessels. In contrast with digital intravenous subtraction angiography it has the advantage of a reliable screening of the ostium and proximal part of the vertebral artery. In the event of a positive test result and the clinical importance of reduction of blood flow through the vertebrobasilar system a conventional contrast arteriographic study is necessary. Now in more experienced hands it is even possible to investigate the distal part of the artery in the bony canal till it loops around the atlas in about $70 \%$ of the cases. If a vertebral artery is found with the duplex scanner, in most cases one is informed about the direction of blood without any special test. A subclavian steal syndrome is very easily and reliably diagnosed. This is also an important advantage above conventional Doppler techniques.

For the detection of an obstructive lesion of $50 \%$ or more and occlusions ultrasonic duplex scanning of the subclavian arteries shows a sensitivity and a specificity of respectively $0.73(11 / 15)$ and 0.91 $(121 / 133)$. In only 6 of the 118 angiographically normal subclavian arteries there was a false positive result. In three of these six cases the turbulent flow in the subclavian artery was probably the result of a marked stenosis of the innominate artery. Similar to the vertebral artery a classification of the degree of stenosis was a problem. One reason was that in vessels with minor wall irregularities it was difficult to differentiate the increased turbulence from eddies as a result of an eccentric placed sample volume in a normal vessel. Another reason was certainly that, depending on local anatomical properties, it was not always possible to place the sample volume near the stenosis. The same problem applied for the investigation of the innominate artery. Especially for these deeper lying vessels a better adjustment of the range of the scan head is very important.

Acknowledgements -We would like to thank J. M. te Riele for preparing this manuscript. This study was supported by the Dutch Heart Foundation.

\section{REFERENCES}

Ackerstaff R. G. A., Eikelboom B. C., Hoeneveld H., Moll F. L. and de Vries A. R. (1982). The accuracy of ultrasonic duplex scanning in carotid artery disease. Clinical Neurology and Neurosurgery 84-4, 211-220.

Brcslau P. J. (1982) Ultrasonic duplex scanning in the evaluation of carotid artery disease. Thesis. Heerlen, The Nethelands.

Büdingen H. J., von Reutern G. M. and Freund H. J. (1977) Diagnosis of cerebrovascular lesions by ultrasonic methods. Int. J. Neurol. 11, 206-218.

Cormier J. M. and Laurian C. (1976) Surgical management of vertebro-basilar insufficiency. J. Cardiovasc. Surg. 17, 205-223.

Fell G., Philips D. J., Chikos P. M., Harley J. D., Thiele B. L., Strandness D. E., Jr. (1981) Ultrasonic duplex scanning for disease of the carotid artery. Circulation 64, 1191-1195.

Hennerici M., Aulich A. and Sandmann W. (1980) Der Wert der Dopplersonographie für Prognose und Therapie schlaganfallgefährdeter Patienten. Angio 3, 151-160.

Hennerici M., Aulich A., Sandmann W. and Freund H. J. (1981) Incidence of asymptomatic extracranial arterial disease. Stroke 12, 750-758.

Hessel S. J., Adams D. F. and Abrams H. L. (1981) Complications of angiography. Radiology 138, 273-281.

Kannel W. B. (1976) Epidemiology of cerebral vascular disease. In Cerebral Arterial Disease (Edited by R. W. Russel), pp. 1-23. Churchill Livingstone, New York.

Keller H. M., Meider W. E. and Kumpe D. A. (1976) Noninvasive angiography for diagnosis of vertebral artery disease using 
Doppler ultrasound (vertebral artery Doppler). Stroke 7, 364-369.

Khodadad G. and McLaurin R. L. (1978) Syndromes of vertebrobasilar insufficiency and their possible surgical treatment. $J$. Fam. Practice 6, 1185-1190.

Knox R. A., Philips D. J., Breslau P. J., Lawrence R., Primozich I., Strandness D. F., Ir. (1982) Empirical findings relating sample volume size to diagnostic accuracy in pulsed Doppler cerebrovascular studies. J. Clin. Ultrasound 10, 227-232.

Naritomi H., Sakai F., Meyer J. S. (1979) Pathogenesis of transient ischemic attacks within the vertebrobasilar arterial system. Arch. Neurol. 36, 121-128.

Reutern von G. M., Büdingen H. J. and Freund H. J. (1976) Dopplersonografische Diagnostiek von Stenosen und Verschlüssen der Vertebralarterien und des Subclavian Steal-syndroms. Arch. Pschiat. Nervenkr. 222, 209-222.

Reutern von G. M. and Pourcelot L. (1978) Cardiac cycledependent alternating flow in vertebral arteries with subclavian artery stenoses. Stroke 9, 229-236.

Rosenthal D., Cossmann D., Ledig C. B. and Callow A. D. (1978)
Results of carotid endarterectomy for vertebrobasilar insufficiency. Arch. Surg. 113, 1361-1364.

Sindermann F. (1967) Krankheitsbild und kollateralkreislauf bei einseitigem und Doppelseitigem Carotisverschluss. J. Neurol. Sci. 5, 9-25.

Sheehan S., Bauer R. B. and Meyer J. S. (1960) Vertebral artery compression in cervical spondylosis. Arteriographic demonstration during life of vertebral artery insufficiency due to rotation and extension of the neck. Neurology 10, 968-986.

Spencer M. P. (1977) Doppler Ultrasonic imaging and noninvasive cerebrovascular evaluation. Int. J. Neurol. 11, 228-242.

Thevenet A. (1979) Chirugie de l'artère vertébrale. In Encyclopédie Médico-Chirurgicale (Paris). S.G.I.M., Les Martres-de-VeyreNo. $76800,43175,3.21 .02$.

White D. N., Ketelaars C. E. J. and Cledgett P. R. (1980) Non-invasive techniques for the recording of vertebral artery flow and their limitations. Ultrasound in Med. \& Biol. 6, 315-327.

Wood C. P. L. and Meire H. B. (1980) A technique for imaging the vertebral artery using pulsed Doppler ultrasound. Ultrasound in Med \& Biol. 6, 329-339. 